\title{
EXISTENCE OF SOLUTIONS FOR A SEMILINEAR ELLIPTIC SYSTEM
}

\author{
Mohamed Benrhouma ${ }^{1}$
}

\begin{abstract}
This paper deals with the existence of solutions to the following system:

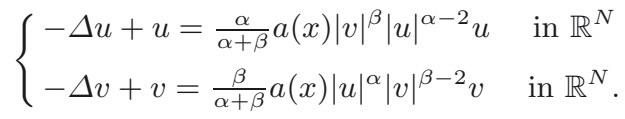

With the help of the Nehari manifold and the linking theorem, we prove the existence of at least two nontrivial solutions. One of them is positive. Our main tools are the concentration-compactness principle and the Ekeland's variational principle.
\end{abstract}

Mathematics Subject Classification. 35J45, 35J50, 35J60.

Received September 14, 2011. Revised April 4, 2012.

Published online February 15, 2013.

\section{INTRODUCTION}

In this paper, we are concerned with the existence of solutions of the following system:

$$
\begin{cases}-\Delta u+u=\frac{\alpha}{\alpha+\beta} a(x)|v|^{\beta}|u|^{\alpha-2} u & \text { in } \mathbb{R}^{N} \\ -\Delta v+v=\frac{\beta}{\alpha+\beta} a(x)|u|^{\alpha}|v|^{\beta-2} v & \text { in } \mathbb{R}^{N} .\end{cases}
$$

Our main hypotheses are cited below:

$\left(H_{1}\right) \quad N>2, \quad 1<\alpha<\frac{N}{N-2}, \quad 1<\beta<\frac{N}{N-2}, \quad$ and $\quad \max (\alpha, \beta) \leq 2$.

$\left(H_{2}\right) \quad a \in L^{\infty}\left(\mathbb{R}^{N}\right), a \geq 0, a \neq 0$ and $\lim _{|x| \rightarrow \infty} a(x)=0$.

The problem of existence of solutions for the semilinear elliptic system

$$
\left\{\begin{array}{l}
-\Delta u=f(x, u, v) \\
-\Delta v=g(x, u, v)
\end{array} \quad x \in \Omega,\right.
$$

Keywords and phrases. Semilinear elliptic systems, Nehari manifold, concentration-compactness principle, variational methods.

1 Mathematics Department, Sciences Faculty of Monastir, 5019 Monastir, Tunisia. brhouma06@yahoo.fr 
with various boundary conditions as well as a bounded domain has been studied by many authors during last decades. we can see for instance $[1,5,7,8,12,19,21]$ and the references therein. A good survey on the existence and nonexistence results for (1.2) with $f(x, u, v)=v^{p}$ and $g(x, u, v)=u^{p}$ can be found by Serrin and Zou in [19]. By the contrast, it seems to us that there are few results are known in $\mathbb{R}^{N}$, we can quote $[3,4,9,11,13,14]$ in which the authors treated the special case of system (1.2),

$$
f(x, u, v)=-u+F(x, v) \quad \text { and } \quad g(x, u, v)=-v+G(x, u) .
$$

De Figueiredo and Yang [9] proved the existence of a strong radial solution pair of (1.2) and the exponential decay of the solution under the assumptions that $f(x, t)$ and $g(x, t)$ are radially symmetric with respect to $x$. Gongbao and Chunhua [11] proved the existence of at least one positive solution of (1.2) by using a linking theorem and the concentration-compactness principle. Li and Yang [14] obtained the existence of a positive solution pair to $(1.2)$ where $f(x, t), g(x, t)$ are asymptotically linear. In the case,

$$
f(x, u, v)=-a(x) u+F_{u}(x, u, v) \quad \text { and } \quad g(x, u, v)=-b(x) v+F_{v}(x, u, v),
$$

among other results, D.G. Costa [6] proved the existence of a nontrivial solution of (1.2) by using the Generalized Mountain Pass theorem.

In the present work, we are interested in finding the existence of two nontrivial solutions of (1.1) and one of them is positive (i.e.: $(u, v)$ is a positive if $u>0$ and $v>0$ a.e.).

The main difficulties to deal with the system (1.1) consist in at least two aspects. In the first hand, on the contrary of the most of the works cited above, the nonlinear part of the system (1.1) $(f(x, u, v), g(x, u, v))$ depends at the same time on $u$ and $v$. In the second hand, as $\mathbb{R}^{N}$ is translation invariant, the Sobolev compact imbedding does not hold on $\mathbb{R}^{N}$. To overcome these difficulties, we study the minimization problem of the appropriate functional on the Nehari manifold [17] corresponding to (1.1). Our mains tools are the concentrationcompactness principle due to Lions $[15,16]$ and the Ekeland's variational principle [10]. Our main result is the following.

Theorem 1.1. Assume $\left(H_{1}\right)$ and $\left(H_{2}\right)$ hold. Then, the system (1.1) possesses at least two nontrivial solutions. One of them is positive.

We organise this paper into four sections. In Section 2, we give some preliminaries and useful results. In Section 3, we prove the existence of a first solution by minimization problem on the Nehari manifold. the compactness of this problem is solved in three steps while applying the cencentration-compactness principle and a major difficulty arise to show the no vanishing of the minimizing sequence. In the last section, we show the existence of a second solution by using the linking theorem $[2,18,20]$ applicable to an auxiliary problem depending of the first solution.

\section{Preliminary}

Let $H=H^{1}\left(\mathbb{R}^{N}\right) \times H^{1}\left(\mathbb{R}^{N}\right)$ and define the inner product $(u, v) \in H$ and $(\varphi, \psi) \in H$ by

$$
\langle(u, v),(\varphi, \psi)\rangle_{H}=\int_{\mathbb{R}^{N}}(\nabla u \nabla \varphi+u \varphi) \mathrm{d} x+\int_{\mathbb{R}^{N}}(\nabla v \nabla \psi+v \psi) \mathrm{d} x
$$

and for $z=(u, v) \in H$, the norm of $z$ is given by

$$
\|z\|=\left(\langle(u, v),(u, v)\rangle_{H}\right)^{\frac{1}{2}} .
$$

It easy to see that $\left(H,\langle., .\rangle_{H}\right)$ is a Hilbert space. 
We say that $(u, v)$ is a weak solution pair of the problem (1.1), if $(u, v) \in H$ and

$$
\begin{array}{r}
\int_{\mathbb{R}^{N}}(\nabla u \nabla \varphi+u \varphi+\nabla v \nabla \psi+v \psi) \mathrm{d} x-\frac{\alpha}{\alpha+\beta} \int_{\mathbb{R}^{N}} a(x)|v|^{\beta}|u|^{\alpha-2} u \varphi \mathrm{d} x-\frac{\beta}{\alpha+\beta} \int_{\mathbb{R}^{N}} a(x)|u|^{\alpha}|v|^{\beta-2} v \psi \mathrm{d} x=0 \\
\forall(\varphi, \psi) \in C_{c}^{\infty}\left(\mathbb{R}^{N}\right) \times C_{c}^{\infty}\left(\mathbb{R}^{N}\right) .
\end{array}
$$

It is clear that $(0,0)$ is a solution of (1.1). We are interested in getting nontrivial solutions to (1.1) which correspond to critical points of the following functional,

$$
I(u, v)=\frac{1}{2} \int_{\mathbb{R}^{N}}\left(|\nabla u|^{2}+u^{2}+|\nabla v|^{2}+v^{2}\right) \mathrm{d} x-\frac{1}{\alpha+\beta} \int_{\mathbb{R}^{N}} a(x)|u|^{\alpha}|v|^{\beta} \mathrm{d} x .
$$

We have $I \in C^{1}(H, \mathbb{R})$ and any critical points of $I$ on $H$ is a weak solution pair of (1.1). The functional $I$ is not bounded neither above nor below on $H$ so we introduce the Nehari manifold.

$$
N=\left\{(u, v) \in H \backslash\{(0,0)\},\left\langle I^{\prime}(u, v),(u, v)\right\rangle=0\right\}
$$

and set

$$
F(u, v)=\left\langle I^{\prime}(u, v),(u, v)\right\rangle=\|(u, v)\|^{2}-\int_{\mathbb{R}^{N}} a(x)|u|^{\alpha}|v|^{\beta} \mathrm{d} x .
$$

It is clear that $F \in C^{1}(H, \mathbb{R})$ and observe that $F^{\prime}(u, v) \neq 0$ for any $(u, v) \in N$.

At first, we prove that the Nehari manifold $N$ is not empty.

Lemma 2.1. $N \neq \emptyset$

Proof. Let $(u, v) \in H$ such that $u>0$ and $v>0$ a.e. in $\mathbb{R}^{N}$. Then,

$$
\int_{\mathbb{R}^{N}} a(x)|u|^{\alpha}|v|^{\beta} \mathrm{d} x>0
$$

Consider the following functional,

$$
\begin{aligned}
\phi_{u, v}(t) & =\frac{t^{2}}{2} \int_{\mathbb{R}^{N}}\left(|\nabla u|^{2}+u^{2}+|\nabla v|^{2}+v^{2}\right) \mathrm{d} x-\frac{t^{\alpha+\beta}}{\alpha+\beta} \int_{\mathbb{R}^{N}} a(x)|u|^{\alpha}|v|^{\beta} \mathrm{d} x \\
& =I(t u, t v)
\end{aligned}
$$

where $(u, v)$ is fixed above. We have $\phi_{u, v}^{\prime}(t)=\left\langle I^{\prime}(t u, t v),(u, v)\right\rangle$, then to prove that $N \neq \emptyset$, we look for critical points of $\phi_{u, v}$. We have $\phi_{u, v}^{\prime}(0)=0, \phi_{u, v}^{\prime}(t)>0$ for $t$ small enough and $\lim _{t \rightarrow+\infty} \phi_{u, v}^{\prime}(t)=-\infty$. Thus, there exists $t_{0}>0$ such that $\phi_{u, v}^{\prime}\left(t_{0}\right)=0$, it follows that $\left(t_{0} u, t_{0} v\right) \in N$.

Now, we give some properties for the Nehari manifold $N$.

Lemma 2.2. $\left(I\left(u_{n}, v_{n}\right)\right)$ is bounded, if and only if $\left(u_{n}, v_{n}\right)$ is bounded in $H, \forall\left(u_{n}, v_{n}\right) \in N$.

Proof. Let $\left(u_{n}, v_{n}\right)$ be a sequence of $N$, we have

$$
\begin{aligned}
I\left(u_{n}, v_{n}\right) & =\frac{1}{2}\left\|\left(u_{n}, v_{n}\right)\right\|^{2}-\frac{1}{\alpha+\beta} \int_{\mathbb{R}^{N}} a(x)\left|u_{n}\right|^{\alpha}\left|v_{n}\right|^{\beta} \mathrm{d} x \\
& =\frac{\alpha+\beta-2}{2(\alpha+\beta)}\left\|\left(u_{n}, v_{n}\right)\right\|^{2} .
\end{aligned}
$$

This gives the wanted result. 


\section{EXISTENCE OF THE FIRST SOLUTION}

In this section, we show the existence of a solution of (1.1) which is a local minimizer for $I$ on $N$.

Consider the Nehari minimization problem

$$
d=\inf \{I(u, v) ;(u, v) \in N\} .
$$

The following lemma is needed to study the existence of a minimum for the functional $I$ on $N$.

Lemma 3.1. $d=\inf \{I(u, v) ;(u, v) \in N\}>0$.

Proof. Let $(u, v) \in N$, we have

$$
I(u, v)=\frac{\alpha+\beta-2}{2(\alpha+\beta)}\|(u, v)\|^{2}>0 .
$$

Which implies that $d \geq 0$.

If $d=0$, then there exists $\left(u_{n}, v_{n}\right) \in N$ such that $I\left(u_{n}, v_{n}\right) \rightarrow 0$. We conclude, by (2.1), that

$$
\left\|\left(u_{n}, v_{n}\right)\right\| \rightarrow 0
$$

On the other hand, we have

$$
\left\|\left(u_{n}, v_{n}\right)\right\|^{2}-\int_{\mathbb{R}^{N}} a(x)\left|u_{n}\right|^{\alpha}\left|v_{n}\right|^{\beta} \mathrm{d} x=0 .
$$

Dividing (3.3) by $\left\|\left(u_{n}, v_{n}\right)\right\|^{2}$, we obtain

$$
1-\frac{1}{\left\|\left(u_{n}, v_{n}\right)\right\|^{2}} \int_{\mathbb{R}^{N}} a(x)\left|u_{n}\right|^{\alpha}\left|v_{n}\right|^{\beta} \mathrm{d} x=0 .
$$

Also, by (3.2), we get

$$
\begin{aligned}
\left.\left|\frac{1}{\left\|\left(u_{n}, v_{n}\right)\right\|^{2}} \int_{\mathbb{R}^{N}} a(x)\right| u_{n}\right|^{\alpha}\left|v_{n}\right|^{\beta} \mathrm{d} x \mid & \leq \frac{1}{\left\|\left(u_{n}, v_{n}\right)\right\|^{2}}|a|_{\infty}\left\|u_{n}\right\|_{2 \alpha}^{\alpha}\left\|v_{n}\right\|_{2 \beta}^{\beta} \\
& \leq \frac{1}{\left\|\left(u_{n}, v_{n}\right)\right\|^{2}} S_{1}^{\alpha} S_{2}^{\beta}|a|_{\infty}\left\|\left(u_{n}, v_{n}\right)\right\|^{\alpha+\beta} \\
& \leq S_{1}^{\alpha} S_{2}^{\beta}|a|_{\infty}\left\|\left(u_{n}, v_{n}\right)\right\|^{\alpha+\beta-2} \rightarrow 0,
\end{aligned}
$$

where $S_{1}$ (respectively $S_{2}$ ) is the best Sobolev constant for the embedding of $H^{1}\left(\mathbb{R}^{N}\right)$ in $L^{2 \alpha}\left(\mathbb{R}^{N}\right.$ ) (respectively $\left.L^{2 \beta}\left(\mathbb{R}^{N}\right)\right)$.

Using (3.5) and passing to the limit in (3.4), we obtain a contradiction. this achieves the proof of Lemma 3.1.

Remark 3.2. We can follow the arguments used in the proof of Lemma 3.1 for proving that the Nehari manifold $N$ is a complete space. So, we can apply the Ekeland's variational principle [10] to the Nehari minimization problem (3.1) which provides the existence of $\left(u_{n}, v_{n}\right) \in N$ and $\left(\lambda_{n}\right) \in \mathbb{R}$ such that

$$
I\left(u_{n}, v_{n}\right) \rightarrow d \text { and } I^{\prime}\left(u_{n}, v_{n}\right)-\lambda_{n} F^{\prime}\left(u_{n}, v_{n}\right) \rightarrow 0 \text { in } H^{\prime}
$$

where $H^{\prime}$ is the dual space of $H$.

$\left(u_{n}, v_{n}\right)$ is called a (PS) sequence of the functional $I$ on $N$. 
Lemma 3.3. Any (PS) sequence of the functional $I$ on $N$ is a $(P S)$ sequence of the functional $I$ on $H$.

Proof. Let $\left(u_{n}, v_{n}\right) \in N$ and $\left(\lambda_{n}\right) \in \mathbb{R}$ be the sequences as in the Remark 3.2. By Lemma 2.2, $\left(u_{n}, v_{n}\right)$ is bounded in $H$. Consequently, by (3.6), we obtain that

$$
\left\langle I^{\prime}\left(u_{n}, v_{n}\right),\left(u_{n}, v_{n}\right)\right\rangle-\lambda_{n}\left\langle F^{\prime}\left(u_{n}, v_{n}\right),\left(u_{n}, v_{n}\right)\right\rangle \rightarrow 0 .
$$

It leads to,

$$
\lambda_{n}\left\langle F^{\prime}\left(u_{n}, v_{n}\right),\left(u_{n}, v_{n}\right)\right\rangle \rightarrow 0
$$

We have

$$
\begin{aligned}
\left\langle F^{\prime}\left(u_{n}, v_{n}\right),\left(u_{n}, v_{n}\right)\right\rangle & =\left\|\left(u_{n}, v_{n}\right)\right\|^{2}-(\alpha+\beta-1) \int_{\mathbb{R}^{N}} a(x)\left|u_{n}\right|^{\alpha}\left|v_{n}\right|^{\beta} \mathrm{d} x \\
& =(2-\alpha-\beta)\left\|\left(u_{n}, v_{n}\right)\right\|^{2} .
\end{aligned}
$$

The sequence $\left(\left\langle F^{\prime}\left(u_{n}, v_{n}\right),\left(u_{n}, v_{n}\right)\right\rangle\right)$ is bounded and doesn't possess a subsequence which is converging to zero. Thus, we conclude, by (3.8), that $\lambda_{n} \rightarrow 0$ and, by (3.6), that

$$
I^{\prime}\left(u_{n}, v_{n}\right) \rightarrow 0 \text { in } H^{\prime} .
$$

This gives the wanted result.

Theorem 3.4. Assume $\left(H_{1}\right)$ and $\left(H_{2}\right)$ hold. Then, the system (1.1) possesses a nontrivial solution pair $(u, v)$ which is positive.

Proof. By Remark 3.2 and Lemma 3.3, there exists $\left(u_{n}, v_{n}\right) \in N$ such that

$$
I\left(u_{n}, v_{n}\right) \rightarrow d \text { and } I^{\prime}\left(u_{n}, v_{n}\right) \rightarrow 0 \text { in } H^{\prime} .
$$

Since $\left(u_{n}, v_{n}\right)$ is bounded in $H$, then there exist $U$ and $V$ in $H^{1}\left(\mathbb{R}^{N}\right)$ such that

$$
u_{n} \rightarrow U \text { and } v_{n} \rightarrow V \text { in } H^{1}\left(\mathbb{R}^{N}\right) .
$$

Let $(\varphi, \psi) \in C_{c}^{\infty}\left(\mathbb{R}^{N}\right) \times C_{c}^{\infty}\left(\mathbb{R}^{N}\right)$, we have

$$
\begin{aligned}
\left\langle I^{\prime}\left(u_{n}, v_{n}\right),(\varphi, \psi)\right\rangle= & \int_{\mathbb{R}^{N}}\left(\nabla u_{n} \nabla \varphi+u_{n} \varphi+\nabla v_{n} \nabla \psi+v_{n} \psi\right) \mathrm{d} x-\frac{\alpha}{\alpha+\beta} \int_{\mathbb{R}^{N}} a(x)\left|v_{n}\right|^{\beta}\left|u_{n}\right|^{\alpha-2} u_{n} \varphi \mathrm{d} x \\
& -\frac{\beta}{\alpha+\beta} \int_{\mathbb{R}^{N}} a(x)\left|u_{n}\right|^{\alpha}\left|v_{n}\right|^{\beta-2} v_{n} \psi \mathrm{d} x \rightarrow 0 .
\end{aligned}
$$

By the weak convergence of $u_{n}$ and $v_{n}$ to $U$ and $V$ in $H^{1}\left(\mathbb{R}^{N}\right)$ respectively, we get

$$
\int_{\mathbb{R}^{N}}\left(\nabla u_{n} \nabla \varphi+u_{n} \varphi+\nabla v_{n} \nabla \psi+v_{n} \psi\right) \mathrm{d} x \rightarrow \int_{\mathbb{R}^{N}}(\nabla U \nabla \varphi+U \varphi+\nabla V \nabla \psi+V \psi) \mathrm{d} x .
$$

Since $u_{n} \rightarrow U$ in $L^{2 \alpha}(\operatorname{supp}(\varphi))$ and $v_{n} \rightarrow V$ in $L^{2 \beta}(\operatorname{supp}(\varphi))$, then there exist $h \in L^{2 \alpha}(\operatorname{supp}(\varphi))$, $\xi \in L^{2 \beta}(\operatorname{supp}(\varphi))$ and up to a subsequence,

$$
\begin{aligned}
& a(x)\left|v_{n}\right|^{\beta}\left|u_{n}\right|^{\alpha-2} u_{n} \varphi \rightarrow a(x)|V|^{\beta}|U|^{\alpha-2} U \varphi \text { a.e. } \\
& |a|\left|v_{n}\right|^{\beta}\left|u_{n}\right|^{\alpha-1}|\varphi| \leq|a|_{\infty}|\xi|^{\beta}|h|^{\alpha-1}|\varphi| \text { in } L^{1} .
\end{aligned}
$$


By the virtue of Lebesgue's dominated convergence theorem, we deduce that

$$
\int_{\mathbb{R}^{N}} a(x)\left|v_{n}\right|^{\beta}\left|u_{n}\right|^{\alpha-2} u_{n} \varphi \mathrm{d} x \rightarrow \int_{\mathbb{R}^{N}} a(x)|V|^{\beta}|U|^{\alpha-2} U \varphi \mathrm{d} x .
$$

Also, by the same argument used above, we obtain

$$
\int_{\mathbb{R}^{N}} a(x)\left|u_{n}\right|^{\alpha}\left|v_{n}\right|^{\beta-2} v_{n} \psi \mathrm{d} x \rightarrow \int_{\mathbb{R}^{N}} a(x)|U|^{\alpha}|V|^{\beta-2} V \psi \mathrm{d} x .
$$

Combining (3.9)-(3.12), we get

$$
\left\langle I^{\prime}(U, V),(\varphi, \psi)\right\rangle=0, \quad \forall(\varphi, \psi) \in C_{c}^{\infty}\left(\mathbb{R}^{N}\right) \times C_{c}^{\infty}\left(\mathbb{R}^{N}\right)
$$

and $(U, V)$ is a solution of the problem (1.1).

Now, we prove that $d=I(U, V)$.

We have $u_{n} \rightarrow U$ and $v_{n} \rightarrow V$ in $H^{1}\left(\mathbb{R}^{N}\right)$ respectively, then $\|(U, V)\| \leq \underline{\lim }\left\|\left(u_{n}, v_{n}\right)\right\|$. We distinguish two cases:

$(\bullet)$ Compactness: $\|(U, V)\|=\lim _{n \rightarrow \infty}\left\|\left(u_{n}, v_{n}\right)\right\|$ (up to a subsequence).

It follows that, $\left(u_{n}, v_{n}\right) \rightarrow(U, V)$ in $H$ and by the continuity of $I$, we get $I(U, V)=d$.

$(\bullet \bullet)$ Dichotomy: $\|(U, V)\|<\lim _{n \rightarrow \infty}\left\|\left(u_{n}, v_{n}\right)\right\|$.

We should prove, in two steps, that this case doesn't occur. Set $w_{n}=u_{n}-U$ and $s_{n}=v_{n}-V$, we have $w_{n} \rightarrow 0$ and $s_{n} \rightarrow 0$ in $H^{1}\left(\mathbb{R}^{N}\right)$.

Step 1. There exists $\left(y_{n}^{1}\right) \subset \mathbb{R}^{N}$ such that

$$
w_{n}\left(.+y_{n}^{1}\right) \rightarrow W \neq 0 \text { or } s_{n}\left(.+y_{n}^{1}\right) \rightarrow S \neq 0 \text { in } H^{1}\left(\mathbb{R}^{N}\right) .
$$

Proof. Suppose that $\forall\left(y_{n}\right) \subset \mathbb{R}^{N}$, we have

$$
w_{n}\left(.+y_{n}\right) \rightarrow 0 \text { and } s_{n}\left(.+y_{n}\right) \rightarrow 0 \text { in } H^{1}\left(\mathbb{R}^{N}\right) .
$$

Therefore,

$$
\forall R>0 \quad \sup _{y \in \mathbb{R}^{N}} \int_{B(y, R)}\left|w_{n}\right|^{2} \mathrm{~d} x \rightarrow 0 \text { and } \sup _{y \in \mathbb{R}^{N}} \int_{B(y, R)}\left|s_{n}\right|^{2} \mathrm{~d} x \rightarrow 0 .
$$

By arguments due to Lions $[15,16]$, we have

$$
w_{n} \rightarrow 0 \text { and } s_{n} \rightarrow 0 \text { in } L^{q}\left(\mathbb{R}^{N}\right) \quad \forall \quad 2<q<2^{*} .
$$

First, observe that there exists $C>0$ such that for any reals $a$ and $b$, we have

$$
|a|^{m}+|b|^{m}-C|a|^{\frac{m}{2}}|b|^{\frac{m}{2}} \leq|a+b|^{m} \leq|a|^{m}+|b|^{m}+C|a|^{\frac{m}{2}}|b|^{\frac{m}{2}}, \quad \forall 0<m \leq 2 .
$$


Replacing $u_{n}$ by $w_{n}+U$ and $v_{n}$ by $s_{n}+V$ in the following expression,

$$
\begin{aligned}
\left\langle I^{\prime}\left(u_{n}, v_{n}\right),\left(u_{n}, v_{n}\right)\right\rangle= & \left\|\left(u_{n}, v_{n}\right)\right\|^{2}-\int_{\mathbb{R}^{N}} a(x)\left|u_{n}\right|^{\alpha}\left|v_{n}\right|^{\beta} \mathrm{d} x \\
\geq & \int_{\mathbb{R}^{N}}\left(\left|\nabla w_{n}\right|^{2}+w_{n}^{2}+|\nabla U|^{2}+U^{2}+\left|\nabla s_{n}\right|^{2}+s_{n}^{2}+|\nabla V|^{2}+V^{2}\right) \mathrm{d} x \\
& +2 \int_{\mathbb{R}^{N}}\left(\nabla w_{n} \nabla U+w_{n} U+\nabla s_{n} \nabla V+s_{n} V\right) \mathrm{d} x \\
& -\int_{\mathbb{R}^{N}} a(x)\left(|U|^{\alpha}+\left|w_{n}\right|^{\alpha}+C|U|^{\frac{\alpha}{2}}\left|w_{n}\right|^{\frac{\alpha}{2}}\right)\left(|V|^{\beta}+\left|s_{n}\right|^{\beta}+C|V|^{\frac{\beta}{2}}\left|s_{n}\right|^{\frac{\beta}{2}}\right) \mathrm{d} x \\
\geq & \int_{\mathbb{R}^{N}}\left(\left|\nabla w_{n}\right|^{2}+w_{n}^{2}+|\nabla U|^{2}+U^{2}+\left|\nabla s_{n}\right|^{2}+s_{n}^{2}+|\nabla V|^{2}+V^{2}\right) \mathrm{d} x \\
& +2 \int_{\mathbb{R}^{N}}\left(\nabla w_{n} \nabla U+w_{n} U+\nabla s_{n} \nabla V+s_{n} V\right) \mathrm{d} x \\
& -\int_{\mathbb{R}^{N}} a(x)|U|^{\alpha}|V|^{\beta} \mathrm{d} x-|a|_{\infty}\|U\|_{2 \alpha}^{\alpha}\left\|s_{n}\right\|_{2 \beta}^{\beta}-C|a|_{\infty}\|U\|_{2 \alpha}^{\alpha}\left\|s_{n}\right\|_{2 \beta}^{\frac{\beta}{2}}\|V\|_{2 \beta}^{\frac{\beta}{2}} \\
& -|a|_{\infty}\left\|w_{n}\right\|_{2 \alpha}^{\alpha}\|V\|_{2 \beta}^{\beta}-|a|_{\infty}\left\|w_{n}\right\|_{2 \alpha}^{\alpha}\left\|s_{n}\right\|_{2 \beta}^{\beta}-C|a|_{\infty}\left\|w_{n}\right\|_{2 \alpha}^{\alpha}\left\|s_{n}\right\|_{2 \beta}^{\frac{\beta}{2}}\|V\|_{2 \beta}^{\frac{\beta}{2}} \\
& -C|a|_{\infty}\left\|w_{n}\right\|_{2 \alpha}^{\frac{\alpha}{2}}\|U\|_{2 \alpha}^{\frac{\alpha}{2}}\left\|s_{n}\right\|_{2 \beta}^{\beta}-C|a|_{\infty}\left\|w_{n}\right\|_{2 \alpha}^{\frac{\alpha}{2}}\|U\|_{2 \alpha}^{\frac{\alpha}{2}}\|V\|_{2 \beta}^{\beta} \\
& -C^{2}|a|_{\infty}\left\|w_{n}\right\|_{2 \alpha}^{\frac{\alpha}{2}}\|U\|_{2 \alpha}^{\frac{\alpha}{2}}\left\|s_{n}\right\|_{2 \beta}^{\frac{\beta}{2}}\|V\|_{2 \beta}^{\frac{\beta}{2}} .
\end{aligned}
$$

By the weak convergence of $w_{n}$ and $s_{n}$ to 0 in $H^{1}\left(\mathbb{R}^{N}\right)$, we obtain

$$
\int_{\mathbb{R}^{N}}\left(\nabla w_{n} \nabla U+w_{n} U+\nabla s_{n} \nabla V+s_{n} V\right) \mathrm{d} x \rightarrow 0 .
$$

Using (3.13), taking into account that $2<2 \alpha<2^{*}, 2<2 \beta<2^{*}$ and passing to the limit in (3.15), we get

$$
0 \geq \lim _{n \rightarrow \infty} \int_{\mathbb{R}^{N}}\left(\left|\nabla w_{n}\right|^{2}+w_{n}^{2}+\left|\nabla s_{n}\right|^{2}+s_{n}^{2}\right) \mathrm{d} x+\left\langle I^{\prime}(U, V),(U, V)\right\rangle .
$$

So $\left\|\left(w_{n}, s_{n}\right)\right\| \rightarrow 0$, which provides a contradiction. The proof of Step 1 is achieved.

We can suppose, in the following, that $w_{n}\left(.+y_{n}^{1}\right) \rightarrow W \neq 0$ in $H^{1}\left(\mathbb{R}^{N}\right)$.

Step 2. $\left(y_{n}^{1}\right)$ is not bounded.

Proof. Suppose that $\left(y_{n}^{1}\right)$ is bounded, then we can extract a subsequence of $\left(y_{n}^{1}\right)$ also denoted by $\left(y_{n}^{1}\right)$ such that $y_{n}^{1} \rightarrow y$. Let $\varphi \in C_{c}^{\infty}\left(\mathbb{R}^{N}\right)$, since $y_{n}^{1} \rightarrow y$ and $w_{n} \rightarrow 0$ in $H^{1}\left(\mathbb{R}^{N}\right)$, then

$$
\int_{\mathbb{R}^{N}} \varphi\left(x-y_{n}^{1}\right) w_{n}(x) \mathrm{d} x \rightarrow 0 .
$$

On the other hand, we have

$$
\int_{\mathbb{R}^{N}} \varphi\left(x-y_{n}^{1}\right) w_{n}(x) \mathrm{d} x=\int_{\mathbb{R}^{N}} \varphi(x) w_{n}\left(x+y_{n}^{1}\right) \mathrm{d} x \rightarrow \int_{\mathbb{R}^{N}} \varphi(x) W(x) \mathrm{d} x .
$$


Combining these last results, it follows that

$$
\int_{\mathbb{R}^{N}} \varphi(x) W(x) \mathrm{d} x=0, \forall \varphi \in C_{c}^{\infty}\left(\mathbb{R}^{N}\right) .
$$

Hence $W=0$ a.e. in $\mathbb{R}^{N}$ which leads to a contradiction. We conclude that $\left(y_{n}^{1}\right)$ is not bounded.

Conclusion. By Steps 1 and 2 , there exists $\left(y_{n}^{1}\right) \subset \mathbb{R}^{N}$ which is not bounded such that $w_{n}\left(.+y_{n}^{1}\right) \rightarrow W \neq 0$ in $H^{1}\left(\mathbb{R}^{N}\right)$.

Now, we prove that $W=0$ a.e. which leads to conclude that the case of Dichotomy does not occur. Indeed, let $\varphi \in C_{c}^{\infty}\left(\mathbb{R}^{N}\right)$, put $\tilde{\varphi}=\varphi\left(.-y_{n}^{1}\right), \tilde{u}_{n}=u_{n}\left(.+y_{n}^{1}\right), \tilde{a}=a\left(.+y_{n}^{1}\right)$ and $\tilde{v}_{n}=v_{n}\left(.+y_{n}^{1}\right)$. We have

$$
\begin{aligned}
\left\langle I^{\prime}\left(u_{n}, v_{n}\right),(\tilde{\varphi}, 0)\right\rangle & =\int_{\mathbb{R}^{N}}\left(\nabla u_{n} \nabla \tilde{\varphi}+u_{n} \tilde{\varphi}\right) \mathrm{d} x-\frac{\alpha}{\alpha+\beta} \int_{\mathbb{R}^{N}} a(x)\left|u_{n}\right|^{\alpha-2} u_{n}\left|v_{n}\right|^{\beta} \tilde{\varphi} \mathrm{d} x \\
& =\int_{\mathbb{R}^{N}}\left(\nabla \tilde{u}_{n} \nabla \varphi+\tilde{u}_{n} \varphi\right) \mathrm{d} x-\frac{\alpha}{\alpha+\beta} \int_{\mathbb{R}^{N}} \tilde{a}\left|\tilde{u}_{n}\right|^{\alpha-2} \tilde{u}_{n}\left|\tilde{v}_{n}\right|^{\beta} \varphi \mathrm{d} x \rightarrow 0 .
\end{aligned}
$$

Since $\left|\left(y_{n}^{1}\right)\right| \rightarrow+\infty$, then $\tilde{u}_{n} \rightarrow W$ in $H^{1}\left(\mathbb{R}^{N}\right)$. It follows that,

$$
\int_{\mathbb{R}^{N}}\left(\nabla \tilde{u}_{n} \nabla \varphi+\tilde{u}_{n} \varphi\right) \mathrm{d} x \rightarrow \int_{\mathbb{R}^{N}}(\nabla W \nabla \varphi+W \varphi) \mathrm{d} x .
$$

Also, $\left(\tilde{v}_{n}\right)$ is bounded in $H^{1}\left(\mathbb{R}^{N}\right)$ then there exists $R \in H^{1}\left(\mathbb{R}^{N}\right)$ such that up to a subsequence $\tilde{v}_{n} \rightarrow R$ in $L_{l o c}^{q}\left(\mathbb{R}^{N}\right), \forall 1 \leq q<2^{*}$. Also, we have $\tilde{u}_{n} \rightarrow W$ in $L^{2 \alpha}(\operatorname{supp}(\varphi))$. Hence, there exist $h_{1} \in L^{2 \alpha}(\operatorname{supp}(\varphi))$, $h_{2} \in L^{2 \beta}(\operatorname{supp}(\varphi))$ and by $\left(H_{2}\right)$, we have

$$
\begin{aligned}
& \tilde{a}\left|\tilde{u}_{n}\right|^{\alpha-2} \tilde{u}_{n}\left|\tilde{v}_{n}\right|^{\beta} \varphi \rightarrow 0 \text { a.e. } \\
& \left.\left.|\tilde{a}| \tilde{u}_{n}\right|^{\alpha-1}\left|\tilde{v}_{n}\right|^{\beta} \varphi|\leq| a\right|_{\infty}\left|h_{1}\right|^{\alpha-1}\left|h_{2}\right|^{\beta}|\varphi| \in L^{1} .
\end{aligned}
$$

By the virtue of Lebesgue's dominated convergence theorem, we deduce that

$$
\int_{\mathbb{R}^{N}} \tilde{a}\left|\tilde{u}_{n}\right|^{\alpha-2} \tilde{u}_{n}\left|\tilde{v}_{n}\right|^{\beta} \varphi \mathrm{d} x \rightarrow 0 .
$$

Combining (3.17)-(3.19), we obtain

$$
\int_{\mathbb{R}^{N}}(\nabla W \nabla \varphi+W \varphi) \mathrm{d} x=0, \quad \forall \varphi \in C_{c}^{\infty}\left(\mathbb{R}^{N}\right) .
$$

Which implies that $W=0$ a.e.

From Steps 1-2, we conclude that the only possible case is the compactness.

Therefore, there exists $(U, V) \in H$ which is a nontrivial solution of $(1.1)(I(U, V)=d>0)$.

Now, we prove that $(|U|,|V|)$ is a positive solution of (1.1).

We have $\left\langle I^{\prime}(|U|,|V|),(|U|,|V|)\right\rangle=0$ and $(|U|,|V|) \neq(0,0)$ then $(|U|,|V|) \in N$. Furthermore, $I(|U|,|V|)=$ $I(U, V)=d$, it follows that there exists $\lambda \in \mathbb{R}$ such that

$$
I^{\prime}(|U|,|V|)=\lambda F^{\prime}(|U|,|V|) .
$$

Put $(|U|,|V|)$ as a test function in (3.20), we get

$$
\begin{aligned}
\left\langle I^{\prime}(|U|,|V|),(|U|,|V|)\right\rangle & =\lambda\left\langle F^{\prime}(|U|,|V|),(|U|,|V|)\right\rangle \\
& =\lambda(2-\alpha-\beta)\|(U, V)\|^{2}=0 .
\end{aligned}
$$


This gives that $\lambda=0$ and by (3.20), we obtain that $I^{\prime}(|U|,|V|)=0$. Which implies that, $(|U|,|V|)$ is a nonnegative solution of (1.1). Moreover, $|U|$ is a weak nonnegative solution of

$$
-\Delta|U|+|U|=\frac{\alpha}{\alpha+\beta} a(x)|V|^{\beta}|U|^{\alpha-1} \quad \text { in } \mathbb{R}^{N} .
$$

The right-hand side of (3.21) is nonnegative and not equivalently equal to 0 . Then, by the maximum principle, $|U|$ is a weak positive solution of (3.21).

By the same argument used above, $|V|$ is a weak positive solution of

$$
-\Delta|V|+|V|=\frac{\beta}{\alpha+\beta} a(x)|U|^{\alpha}|V|^{\beta-1} \quad \text { in } \mathbb{R}^{N} .
$$

Combining the last results, we conclude that $(|U|,|V|)$ is a positive solution of (1.1).

The proof of Theorem 3.4 is achieved.

\section{Existence OF THE SECOND SOlution}

In this section, we prove the existence of a second solution of the system (1.1) by using the linking theorem. We introduce the following auxiliary problem

$$
\left\{\begin{array}{l}
-\Delta u+u=\frac{\alpha}{\alpha+\beta} a(x)\left(|u+U|^{\alpha-2}(u+U)|v+V|^{\beta}-U^{\alpha-1} V^{\beta}\right) \\
-\Delta v+v=\frac{\beta}{\alpha+\beta} a(x)\left(|u+U|^{\alpha}|v+V|^{\beta-2}(v+V)-U^{\alpha} V^{\beta-1}\right)
\end{array}\right.
$$

where $(U, V)$ is the positive solution of (1.1) given by Theorem 3.4.

Lemma 4.1. If $(u, v)$ is a solution of (4.1), then $(u+U, v+V)$ is a solution of (1.1).

Proof. Let $(u, v)$ be a solution of $(4.1)$ and $(\varphi, \psi) \in C_{c}^{\infty}\left(\mathbb{R}^{N}\right) \times C_{c}^{\infty}\left(\mathbb{R}^{N}\right)$,

$$
\begin{aligned}
& \int_{\mathbb{R}^{N}}(\nabla(u+U) \nabla \varphi+(u+U) \varphi+\nabla(v+V) \nabla \psi+(v+V) \psi) \mathrm{d} x \\
& =\frac{\alpha}{\alpha+\beta} \int_{\mathbb{R}^{N}} a(x)\left(|u+U|^{\alpha-2}(u+U)|v+V|^{\beta}-U^{\alpha-1} V^{\beta}\right) \varphi \mathrm{d} x \\
& \quad+\frac{\beta}{\alpha+\beta} \int_{\mathbb{R}^{N}} a(x)\left(|u+U|^{\alpha}|v+V|^{\beta-2}(v+V)-U^{\alpha} V^{\beta-1}\right) \psi \mathrm{d} x \\
& \quad+\int_{\mathbb{R}^{N}} a(x)\left(\frac{\alpha}{\alpha+\beta} U^{\alpha-1} V^{\beta} \varphi+\frac{\beta}{\alpha+\beta} U^{\alpha} V^{\beta-1} \psi\right) \mathrm{d} x \\
& =\int_{\mathbb{R}^{N}} a(x)\left(\frac{\alpha}{\alpha+\beta}|u+U|^{\alpha-2}(u+U)|v+V|^{\beta} \varphi+\frac{\beta}{\alpha+\beta}|u+U|^{\alpha}|v+V|^{\beta-2}(v+V) \psi\right) \mathrm{d} x .
\end{aligned}
$$

Which gives the desire result.

Consider the following functional,

$$
\begin{aligned}
J(u, v)= & \frac{1}{2}\|(u, v)\|^{2}-\frac{1}{\alpha+\beta} \int_{\mathbb{R}^{N}} a(x)|u+U|^{\alpha}|v+V|^{\beta} \mathrm{d} x \\
& +\frac{\alpha}{\alpha+\beta} \int_{\mathbb{R}^{N}} a(x) U^{\alpha-1} V^{\beta} u \mathrm{~d} x+\frac{\beta}{\alpha+\beta} \int_{\mathbb{R}^{N}} a(x) U^{\alpha} V^{\beta-1} v \mathrm{~d} x .
\end{aligned}
$$

It is clear that $J \in C^{1}(H, \mathbb{R})$ and any critical point of $J$ is a solution of (4.1). 
Lemma 4.2. The functional $J$ satisfies the Palais-Smale condition.

Proof. Let $\left(u_{n}, v_{n}\right)$ be a Palais-Smale sequence of the functional $J$ :

$$
\text { (i.e.: } \left.\left.J\left(u_{n}, v_{n}\right)\right) \text { is bounded and } J^{\prime}\left(u_{n}, v_{n}\right) \rightarrow 0 \text { in } H^{\prime}\right) \text {. }
$$

Since $J\left(u_{n}, v_{n}\right)=I\left(u_{n}+U, v_{n}+V\right)-\frac{1}{2}\|(U, V)\|^{2}$, then $I\left(u_{n}+U, v_{n}+V\right)$ is bounded and

$$
I^{\prime}\left(u_{n}+U, v_{n}+V\right) \rightarrow 0 \text { in } H^{\prime} .
$$

Put $w_{n}=u_{n}+U$ and $s_{n}=v_{n}+V,\left(w_{n}, s_{n}\right)$ is a (PS) sequence of $I$. It suffices to prove that $\left(w_{n}, s_{n}\right)$ is bounded in $H$.

For $n$ large enough, we have $\left|I\left(w_{n}, s_{n}\right)\right|<C_{1}$ and $\left|\left\langle I^{\prime}\left(w_{n}, s_{n}\right),\left(w_{n}, s_{n}\right)\right\rangle\right|<\left\|\left(w_{n}, s_{n}\right)\right\|$. It follows that,

$$
I\left(w_{n}, s_{n}\right)-\frac{1}{\alpha+\beta}\left\langle I^{\prime}\left(w_{n}, s_{n}\right),\left(w_{n}, s_{n}\right)\right\rangle=\frac{\alpha+\beta-2}{2(\alpha+\beta)}\left\|\left(w_{n}, s_{n}\right)\right\|^{2}<C_{1}+\frac{1}{\alpha+\beta}\left\|\left(w_{n}, s_{n}\right)\right\| .
$$

Hence $\left(w_{n}, s_{n}\right)$ is bounded in $H$ which implies that $w_{n} \rightarrow w, s_{n} \rightarrow s$ in $H^{1}\left(\mathbb{R}^{N}\right)$ and

$$
\left\langle I^{\prime}\left(w_{n}, s_{n}\right),\left(w_{n}, s_{n}\right)\right\rangle \rightarrow 0 .
$$

Also, we can follow the same argument used in the proof of Theorem 3.4, to prove that the only possible case is the compactness $\left(i . e .:\left(w_{n}, s_{n}\right) \rightarrow(w, s)\right.$ in $\left.H\right)$ that gives $\left(u_{n}, v_{n}\right) \rightarrow(w-U, s-V)$ in $H$. This achieved the proof of Lemma 4.2 .

Now, we give a useful remark.

Remark 4.3. Let $(U, V)$ be the positive solution of (1.1) given by Theorem 3.4 and $(\varphi, \psi) \in C_{c}^{\infty}\left(\mathbb{R}^{N}\right) \times$ $C_{c}^{\infty}\left(\mathbb{R}^{N}\right)$. We have

$$
\left\langle J^{\prime}(U, V),(\varphi, \psi)\right\rangle=\left(2-2^{\alpha+\beta-1}\right) \int_{\mathbb{R}^{N}} a(x) U^{\alpha-1} V^{\beta-1}\left(\frac{\alpha}{\alpha+\beta} V \varphi+\frac{\beta}{\alpha+\beta} U \psi\right) \mathrm{d} x .
$$

Subsequently,

$$
\left\langle J^{\prime}(U, V),(U, V)\right\rangle=\left(2-2^{\alpha+\beta-1}\right) \int_{\mathbb{R}^{N}} a(x) U^{\alpha} V^{\beta} \mathrm{d} x=\left(2-2^{\alpha+\beta-1}\right)\|(U, V)\|^{2} \neq 0 .
$$

Consequently there exists $\left(\varphi_{0}, \psi_{0}\right) \in H$ such that $\left\langle J^{\prime}(U, V),\left(\varphi_{0}, \psi_{0}\right)\right\rangle=1$ and

$$
H=\operatorname{Ker} J^{\prime}(U, V) \oplus \mathbb{R}\left(\varphi_{0}, \psi_{0}\right) .
$$

Where

$$
\operatorname{Ker} J^{\prime}(U, V)=\left\{(\varphi, \psi) \in H,\left\langle J^{\prime}(U, V),(\varphi, \psi)\right\rangle=0\right\} .
$$

Set $X_{1}=\operatorname{Ker} J^{\prime}(U, V), X_{2}=\mathbb{R}\left(\varphi_{0}, \psi_{0}\right)$. We can check easily that $Z=\left(\frac{\beta}{\alpha} U,-V\right) \in X_{1}$.

Consider

$$
S=\left\{u \in X_{1},\|u-Z\| \leq R\right\}
$$

and

$$
Q=\left\{u_{2}-\frac{s}{\|Z\|} Z+Z,\|Z\|-r_{1} \leq s \leq\|Z\|+r_{1} ; u_{2} \in X_{2},\left\|u_{2}\right\| \leq r_{2}\right\}
$$


with relative boundary

$$
\partial Q=\left\{u_{2}-\frac{s}{\|Z\|} Z+Z, s \in\left\{\|Z\|-r_{1},\|Z\|+r_{1}\right\} ; \text { or }\left\|u_{2}\right\|=r_{2}\right\} .
$$

Where $r_{1}, r_{2}, R>0$ will be fixed later such that $r_{1}+R<\|Z\|$.

Lemma 4.4. Let $S$ and $Q$ be fixed above, $S$ and $\partial Q$ link.

Proof. Firstly, we have $S \cap \partial Q=\emptyset$ indeed, if $u=u_{2}+\frac{\|Z\|-s}{\|Z\|} Z \in S \cap \partial Q$, then $u_{2}=0$ and $s \in$ $\left\{\|Z\|-r_{1},\|Z\|+r_{1}\right\}$. Which yield two cases:

(1) $u=\frac{r_{1}}{\|Z\|} Z$ and $\|u-Z\|=\|Z\|-r_{1} \leq R$, which is absurd;

(2) $u=-\frac{r_{1}}{\|Z\|} Z$ and $\|u-Z\|=\|Z\|+r_{1} \leq R$, which is absurd.

Secondly, let $\pi: H \rightarrow X_{2}$ denote the projection onto $X_{2}$ and let $h \in C(H, H)$ satisfy $\left.h\right|_{\partial Q}=i d$. We must show that $h(Q) \cap S \neq \emptyset$. For $t \in[0,1], s \in \mathbb{R}, u_{2} \in X_{2}$. Let

$$
h_{t}\left(s, u_{2}\right)=\left(t\|h(u)-\pi(h(u))-Z\|+(1-t) s, t \pi(h(u))+(1-t) u_{2}\right)
$$

where $u=u_{2}+\frac{\|Z\|-s}{\|Z\|} Z$ and $Z$ fixed above. This defines a family of maps $h_{t}: \mathbb{R} \times X_{2} \rightarrow \mathbb{R} \times X_{2}$ depending continuously on $t \in[0,1]$. Moreover, if $u \in \partial Q$, we have

$$
h_{t}\left(s, u_{2}\right)=\left(s, u_{2}\right) \neq(0,0) \quad \text { for all } t \in[0,1] .
$$

Hence, if we identify $Q$ with a subset of $\mathbb{R} \times X_{2}$ via the decomposition $u=u_{2}+\frac{\|Z\|-s}{\|Z\|} Z$, the topological degree $\operatorname{deg}\left(h_{t}, Q, 0\right)$ is well-defined and by homotopy invariance

$$
\operatorname{deg}\left(h_{1}, Q, 0\right)=\operatorname{deg}\left(h_{0}, Q, 0\right)=\operatorname{deg}(i d, Q, 0)=1 .
$$

Thus, there exists $u \in Q$ such that $h_{1}\left(s, u_{2}\right)=(0,0)$, which is equivalent to

$$
\pi(h(u))=0 \quad \text { and } \quad\|h(u)-Z\|=0 .
$$

The proof of Lemma 4.4 is achieved.

Lemma 4.5. Let $S$ and $Q$ be fixed above, we have

$$
\inf _{(u, v) \in S} J(u, v)>\sup _{(u, v) \in \partial Q} J(u, v) .
$$

Proof. Let $(u, v) \in \partial Q$, then there exists $\delta \in \mathbb{R}$ such that

$$
(u, v)=\delta\left(\varphi_{0}, \psi_{0}\right)+\frac{\|Z\|-s}{\|Z\|} Z
$$


where $Z=\left(\frac{\beta}{\alpha} U,-V\right)$ and $\left(\varphi_{0}, \psi_{0}\right)$ is fixed in Remark 4.3. Put $\lambda=\frac{\|Z\|-s}{\|Z\|}$ with $|\lambda|<1$ and using (3.14), we get

$$
\begin{aligned}
J(u, v)= & \left(\delta \varphi_{0}+\lambda \frac{\beta}{\alpha} U, \delta \psi_{0}-\lambda V\right)=\frac{1}{2}\left\|\left(\delta \varphi_{0}+\lambda \frac{\beta}{\alpha} U, \delta \psi_{0}-\lambda V\right)\right\|^{2} \\
& -\frac{1}{\alpha+\beta} \int_{\mathbb{R}^{N}} a(x)\left|\delta \varphi_{0}+\left(\lambda \frac{\beta}{\alpha}+1\right) U\right|^{\alpha}\left|\delta \psi_{0}+(1-\lambda) V\right|^{\beta} \mathrm{d} x+ \\
& \frac{\alpha}{\alpha+\beta} \int_{\mathbb{R}^{N}} a(x) U^{\alpha-1} V^{\beta}\left(\delta \varphi_{0}+\lambda \frac{\beta}{\alpha} U\right) \mathrm{d} x+\frac{\beta}{\alpha+\beta} \int_{\mathbb{R}^{N}} a(x) U^{\alpha} V^{\beta-1}\left(\delta \psi_{0}-\lambda V\right) \mathrm{d} x \\
\leq & \frac{\delta^{2}}{2}\left\|\left(\varphi_{0}, \psi_{0}\right)\right\|^{2}+\frac{\lambda^{2}}{2}\left\|\left(\frac{\beta}{\alpha} U,-V\right)\right\|^{2}+\delta \lambda \int_{\mathbb{R}^{N}}\left(\frac{\beta}{\alpha}\left(\nabla U \nabla \varphi_{0}+U \varphi_{0}\right)-\left(\nabla V \nabla \psi_{0}+V \psi_{0}\right)\right) \mathrm{d} x \\
& -\left|\left(1+\lambda \frac{\beta}{\alpha}\right)\right|^{\alpha}|(1-\lambda)|^{\beta} \frac{1}{\alpha+\beta} \int_{\mathbb{R}^{N}} a(x) U^{\alpha} V^{\beta} \mathrm{d} x+o(|\delta|,|\lambda|) \\
& +\delta\left(\frac{\alpha}{\alpha+\beta} \int_{\mathbb{R}^{N}} a(x) U^{\alpha-1} V^{\beta} \varphi_{0} \mathrm{~d} x+\frac{\beta}{\alpha+\beta} \int_{\mathbb{R}^{N}} a(x) U^{\alpha} V^{\beta-1} \psi_{0} \mathrm{~d} x\right) \\
\leq & \frac{\delta^{2}}{2}\left\|\left(\varphi_{0}, \psi_{0}\right)\right\|^{2}+\frac{\lambda^{2}}{2}\left\|\left(\frac{\beta}{\alpha} U,-V\right)\right\|^{2}+|\delta|\left(\int_{\mathbb{R}^{N}} a(x) U^{\alpha-1} V^{\beta-1}\left(V\left|\varphi_{0}\right|+\frac{2 \beta}{\alpha+\beta} U\left|\psi_{0}\right|\right) \mathrm{d} x\right) \\
& -\left|\left(1+\lambda \frac{\beta}{\alpha}\right)\right|^{\alpha}|(1-\lambda)|^{\beta} \frac{1}{\alpha+\beta} \int_{\mathbb{R}^{N}} a(x) U^{\alpha} V^{\beta} \mathrm{d} x+o(|\delta|,|\lambda|)
\end{aligned}
$$

where

$$
\lim _{|(|\delta|,|\lambda|)| \rightarrow 0} o(|\delta|,|\lambda|)=0 .
$$

It follows that for $r_{1}^{\prime}$ and $r_{2}^{\prime}$ small enough such that $|\delta|<r_{2}^{\prime}$ and $|\lambda|<r_{1}^{\prime}$, we obtain that

$$
J(u, v) \leq 0, \quad \forall(u, v) \in \partial Q .
$$

We choose $r_{1}<r_{1}^{\prime}\|Z\| \quad$ and $\quad r_{2}<r_{2}^{\prime}\left\|\left(\varphi_{0}, \psi_{0}\right)\right\|$.

On the other hand, let $(u, v) \in X_{1}$. We get, by (4.2), that

$$
J(u, v)=\frac{1}{2}\|(u, v)\|^{2}-\frac{1}{\alpha+\beta} \int_{\mathbb{R}^{N}} a(x)|u+U|^{\alpha}|v+V|^{\beta} \mathrm{d} x
$$

and $J(Z)=\frac{1}{2}\|Z\|^{2}$. So, by the continuity of $J$, there exists $R>0$ such that

$$
J(u, v)>\frac{1}{4}\|Z\|^{2}, \quad \forall(u, v) \in \bar{B}_{R}=\{(u, v) \in H,\|(u, v)-Z\| \leq R\} .
$$

Choosing $R$ small enough such that $r_{1}+R<\|Z\|$ and $S=\bar{B}_{R} \cap X_{1}$.

As desired by (4.3) and (4.4).

Now, we give the main result of this section by applying the linking theorem.

Theorem 4.6. There exists a second nontrivial solution of the system (1.1).

Proof. Let $S$ and $Q$ be fixed in Remark 4.3. Recalling Lemmas 4.2, 4.4 and 4.5, we see that the assumptions of linking theorem are satisfied. Thus $J$ admits a nontrivial critical point $\left(U_{1}, V_{1}\right)$ such that $J\left(U_{1}, V_{1}\right) \geq \frac{1}{4}\|Z\|^{2}$. By Lemma 4.1, $\left(U_{1}+U, V_{1}+V\right)$ is a second solution of (1.1). Furthermore, we have $I\left(U_{1}+U, V_{1}+V\right)=J\left(U_{1}, V_{1}\right)+\frac{1}{2}\|(U, V)\|^{2}>0$ then $\left(U_{1}+U, V_{1}+V\right) \neq(0,0)$.

The proof of lemma 4.6 is achieved. 


\section{REFERENCES}

[1] Y. An, Uniqueness of positive solutions for a class of elliptic systems. J. Math. Anal. Appl. 322 (2006) $1071-1082$.

[2] V. Benci, Some critical point theorems and applications. Commun. Pure Appl. Math. 33 (1980) 147-172.

[3] J. Busca and B. Sirakov, Symmetry results for semilinear elliptic systems in the whole space. J. Differ. Equ. 163 (2000) 41-56.

[4] K.-J. Chen, Multiplicity for strongly indefinite semilinear elliptic system. Nonlinear Anal. 72 (2010) 806-821.

[5] P. Clement, D.G. Figuereido and E. Mitidieri, Positive solutions of semilinear elliptic systems. Commun. Partial Differ. Equ. 17 (1992) 923-940.

[6] D.G. Costa, On a class of elliptic systems in $\mathbb{R}^{N}$. Electron. J. Differ. Equ. 7 (1994) 1-14.

[7] R. Cui, Y. Wang and J. Shi, Uniqueness of the positive solution for a class of semilinear elliptic systems. Nonlinear Anal. 67 (2007) $1710-1714$.

[8] R. Dalmasso, Existence and uniqueness of positive solutions of semilinear elliptic systems. Nonlinear Anal. 39 (2000) $559-568$.

[9] D.G. De Finueirdo and J.F. Yang, Decay, symmetry and existence of solutions of semilinear elliptic systems. Nonlinear Anal. 33 (1998) 211-234.

[10] I. Ekeland, On the variational principle. J. Math. Anal. Appl. 47 (1974) 324-353.

[11] L. Gongbao and W. Chunhua, The existence of nontrivial solutions to a semilinear elliptic system on $\mathbb{R}^{N}$ without the Ambrosetti-Rabinowitz condition. Acta Math. Sci. B 30 (2010) 1917-1936.

[12] D.D. Hai, Uniqueness of positive solutions for semilinear elliptic systems. J. Math. Anal. Appl. 313 (2006) $761-767$.

[13] A.V. Lair and A.W. Wood, Existence of entire large positive solutions of semilinear elliptic systems, J. Differ. Equ. 164 (2000) 380-394.

[14] G.B. Li and J.F. Yang, Asymptotically linear elliptic systems. Commun. Partial Differ. Equ. 29 (2004) $925-954$.

[15] P.L. Lions, the concentration-compactness principle in the calculus of variations. The locally compact case, part 1. Ann. Inst. Henri Poincaré, Anal. Non Linéaire 1 (1984) 109-145.

[16] P.L. Lions, the concentration-compactness principle in the calculus of variations. The locally compact case, part 2. Ann. Inst. Henri Poincaré, Anal. Non Linéaire 2 (1984) 223-283.

[17] Z. Nehari, On a class of nonlinear second-order differential equations. Trans. Amer. Math. Soc. 95 (1960) 101-123.

[18] W.-M. Ni, Some minimax principles and their applications in nonlinear elliptic equations. J. Anal. Math. 37 (1980) $248-275$.

[19] P.H. Rabinowitz, Some critical point theorems and applications to semilinear elliptic partial differential equations. Ann. Scuola Norm. Sup. Pisa Cl. Sci. 5 (1978) 215-223.

[20] J. Serrin and H. Zou, Nonexistence of positive solutions of Lane-Emden systems. Differ. Integral Equ. 9 (1996) 635-653.

[21] T.-F. Wu, The Nehari manifold for a semilinear elliptic system involving sign-changing weight functions. Nonlinear Anal. 68 (2008) 1733-1745. 